$\mathbb{T}$ periodica polytechnica

Civil Engineering

$56 / 2(2012) 167+173$

doi: 10.3311/pp.ci.2012-2.03

web: http://www.pp.bme.hu/ci

(c) Periodica Polytechnica 2012

RESEARCH ARTICLE

\section{The sensitivity of the flutter derivatives and the flutter speed to the eccentricity of the cross section}

\author{
Mátyás Hunyadi / István Hegedûs
}

Received 2011-10-26, revised 2012-02-23, accepted 2012-10-11

\begin{abstract}
The flutter instability analysis of a bridge deck is based on flutter derivatives determined by wind tunnel tests on a section model having two degrees of freedom, heave and pitch (hereafter referred to as the heave-pitch model). The imperfections and the eccentricity that arise during the forced sinusoidal vibration of the section yield erroneous derivatives. This paper studies the relationship between these errors and the imperfections. Rotational excitations around two eccentric axes (hereafter referred to as the pitch-pitch model) of the section model show that the determined derivatives are less error-prone to imperfections. Determining the derivatives, like angular speed flutter derivative $A_{2}^{*}$ for the aeroelastic torsion moment, gives a more accurate value, so the flutter instability analysis yields a more accurate estimate of the flutter wind speed. Numerical values are presented for the case of a thin airfoil and a bluff bridge cross section.
\end{abstract}

\section{Keywords}

Flutter; 2DOF; Pitch-pitch section model

\section{Mátyás Hunyadi}

Department of Structural Engineering, BME, Múegyetem rkp. 3. Budapest, H1111, Hungary

e-mail: hunyadi@vbt.bme.hu

\section{István Hegedús}

Department of Structural Engineering, BME, Múegyetem rkp. 3. Budapest, H1111, Hungary

\section{Introduction}

The considerable interaction of aerodynamic forces and structural motions is called aeroelasticity. Flutter refers to the aeroelastic phenomenon where structural motions become oscillatory with amplitude monotonically increasing in time, which can even lead to the collapse of the structure (in the wide signification of flutter [1]). Flutter instability analysis of a bridge deck begins with wind tunnel investigation of flutter derivatives. These derivatives are used in both simplified and complex verification methods to determine the structure's aeroelastic behaviour and the flutter wind speed, thus the accuracy of the determined derivatives is primordial. This raises the question of the accuracy of these terms, especially with regard to model errors. Nowadays computational fluid dynamics (CFD) simulations tend to give acceptable results [8] and, in the near future, it seems to surmount the issues present in wind tunnel tests and will be the preferred solution to the flutter analysis.

The flutter analysis of a recently built extradosed bridge [4] raised the need to investigate the assumed errors involuntary present in the wind tunnel test driven by a robot arm. The analysis of the effects of some geometric imperfections and eccentricity is the motivation of the present paper.

Some hereafter defined imperfections that can occur in a wind tunnel test, due to geometric constraints and quality of the used test equipment, are studied is this paper. These imperfections have their effects on the determined derivatives, which will thus be prone to be erroneous. Two kinds of errors are analysed for the case of a wind tunnel test set-up with vertical and angular displacement degrees of freedom (hereafter referred to as the heave-pitch model): the eccentricity of the rotation centre and the centre of gravity of the section; and the rotation of the section during vertical displacement excitation. These errors can be related to the geometry of the model and/or to mistaken theoretical assumptions concerning the configuration, hereafter referred to as the imperfections.

The study of the considered imperfections uses a newly developed two degrees of freedom (DOFs) description of the section. This model is used in the analysis of the errors due to the imperfections and in the development of a test configuration where 
the section is forced by two distinct rotations around two given axes (hereafter referred to as the pitch-pitch model). This setup yields new derivatives, from which the derivatives defined to the centre of gravity can be calculated. Imperfections of this model are also studied and presented, which yield derivatives less prone to error.

Eccentric flutter models can also be used for power generation, as is presented in a feasibility study [2].

\section{Description of the pitch-pitch section model}

Consider a symmetric section of a bridge deck on which centre of gravity aeroelastic vertical lift force $L_{h}$ and torsion moment $M_{\alpha}$ per unit length act due to the oncoming wind with speed $U$. The motion of the section subjected to these aeroelastic forces is described by equations [6]

$$
\begin{aligned}
m \ddot{h}+c_{h} \dot{h}+k_{h} h & =L_{h} \\
I_{\alpha} \ddot{\alpha}+c_{\alpha} \dot{\alpha}+k_{\alpha} \alpha & =M_{\alpha}
\end{aligned}
$$

where $h$ and $\alpha$ denote the vertical deflection (heave) and torsional rotation (pitch) of the centre of gravity (c.g.) of the section. The section has $m$ and $I_{\alpha}$ as the mass and mass moment of inertia per unit length, respectively, $c_{h}$ and $c_{\alpha}$ as the viscous damping constants according to the two movements, $k_{h}$ and $k_{\alpha}$ as the stiffness coefficients of the heaving and pitching modes, respectively. Based on analytical theories of Theodorsen [7] and Klöppel et al [5] the aeroelastic forces can be written in a form with force coefficients in function of the motion of the section. These force coefficients have been rewritten in a new form by Scanlan [6]. This latter formulation is used in this paper.

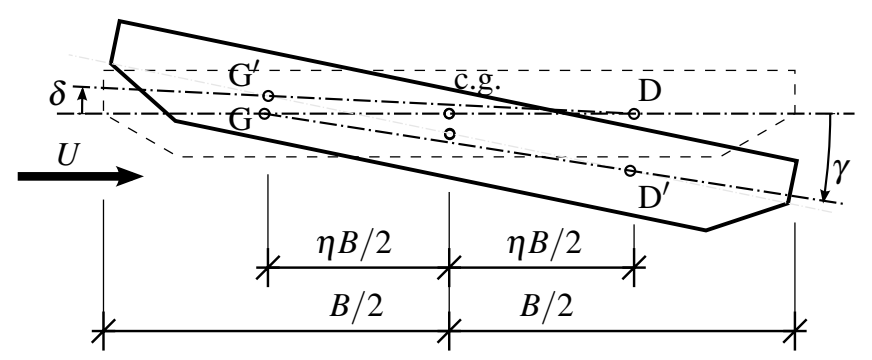

Fig. 1. Section model with two rotational degrees of freedom: $\gamma$ and $\delta$

Consider the same section with two independent rotational degrees of freedom (fig. 11). Define the points G and D in the horizon of the centre of gravity (c.g.) spaced at distance $\eta B / 2$ from the latter to windward and leeward side, respectively, where $B / 2$ denotes the half-width of the section. Denote these same two points by $\mathrm{G}^{\prime}$ and $\mathrm{D}^{\prime}$ in a displaced position of the section. Defining by $\gamma$ the angle of the displaced line $\mathrm{G}-\mathrm{D}^{\prime}$ and by $\delta$ the angle of the line $\mathrm{D}-\mathrm{G}^{\prime}$ to the original horizon results in a well determined description of the displaced position. These two angles will be used as the two degrees of freedom (pitch-pitch DOFs) of the section. There exists a bijective relation between the DOFs of the heave-pitch model and those of the pitch-pitch model supposing small displacements.
The force and moment equilibrium written to the windward rotation axis (denoted by $\mathrm{G}$ ) yields a lift force equivalent to the one acting at c.g. of the section $\left(L_{\gamma}=L_{\alpha}\right)$ and a moment $\left(M_{\gamma}\right)$ which contains the aeroelastic moment $M_{\alpha}$ acting at c.g. and the lift force $L_{h}$ multiplied by the eccentricity. Both relations can be organized to obtain coefficients for the incidences $\gamma$ and $\delta$ and angular speeds $\dot{\gamma}$ and $\dot{\delta}$. These coefficients represent physical phenomena similar to the derivatives of the heave-pitch model, so they can be treated as derivatives and denoted with second indexes $\gamma$ and $\delta$, respectively to the DOF considered. Writing the equilibrium to the leeward axis D gives a relationship for lift force $L_{\delta}$ and moment $M_{\delta}$ analogue to the previously defined ones. These relationships are

$$
\begin{aligned}
L_{\gamma}= & L_{\delta}=\frac{1}{2} \rho U^{2} B\left(K H_{2 \gamma}^{*} \frac{\dot{\gamma} B}{U}+K^{2} H_{3 \gamma}^{*} \gamma+K H_{2 \delta}^{*} \frac{\dot{\delta} B}{U}+K^{2} H_{3 \delta}^{*} \delta\right) \\
M_{\gamma}= & \frac{1}{2} \rho U^{2} B^{2}\left(K A_{2 \gamma}^{*} \frac{\dot{\gamma} B}{U}+K^{2} A_{3 \gamma}^{*} \gamma\right. \\
& \left.+K\left(A_{2 \delta}^{*}+\eta H_{2 \delta}^{*}\right) \frac{\dot{\delta} B}{U}+K^{2}\left(A_{3 \delta}^{*}+\eta H_{3 \delta}^{*}\right) \delta\right) \\
M_{\delta}= & \frac{1}{2} \rho U^{2} B^{2}\left(K\left(A_{2 \gamma}^{*}-\eta H_{2 \gamma}^{*}\right) \frac{\dot{\gamma} B}{U}+K^{2}\left(A_{3 \gamma}^{*}-\eta H_{3 \gamma}^{*}\right) \gamma\right. \\
& \left.+K A_{2 \delta}^{*} \frac{\dot{\delta} B}{U}+K^{2} A_{3 \delta}^{*} \delta\right)
\end{aligned}
$$

where $K=\frac{2 \pi f B}{U}$ is the reduced circular frequency of the motion of the section, $f$ is the frequency of the oscillating motion and $B$ is the width of the section, $U$ is the oncoming wind speed, $\rho$ is the mass density of the air, and, assuming the linearity of the aeroelastic forces, the newly defined $A_{i j}^{*}$ and $H_{i j}^{*}(i=2,3 ; j=$ $\gamma, \delta$ ) derivatives have a bijective relationship with angular and heave derivatives $A_{i}^{*}$ and $H_{i}^{*}(i=1 . .4)$, respectively, defined to the c.g. This relationship is of the form

$$
\begin{aligned}
& A_{2 \gamma}^{*}=\frac{H_{1}^{*} \eta^{2}}{4}+\frac{H_{2}^{*} \eta}{2}+\frac{A_{1}^{*} \eta}{2}+A_{2}^{*} \\
& A_{3 \gamma}^{*}=\frac{H_{3}^{*} \eta}{2}+\frac{H_{4}^{*} \eta^{2}}{4}+A_{3}^{*}+\frac{A_{4}^{*} \eta}{2} \\
& H_{2 \gamma}=\frac{H_{1}^{*} \eta}{2}+H_{2}^{*} \\
& H_{3 \gamma}=H_{3}^{*}+\frac{H_{4}^{*} \eta}{2}
\end{aligned}
$$

and

$$
\begin{aligned}
& A_{2 \delta}^{*}=\frac{H_{1}^{*} \eta^{2}}{4}-\frac{H_{2}^{*} \eta}{2}-\frac{A_{1}^{*} \eta}{2}+A_{2}^{*} \\
& A_{3 \delta}^{*}=-\frac{H_{3}^{*} \eta}{2}+\frac{H_{4}^{*} \eta^{2}}{4}+A_{3}^{*}-\frac{A_{4}^{*} \eta}{2} \\
& H_{2 \delta}=-\frac{H_{1}^{*} \eta}{2}+H_{2}^{*} \\
& H_{3 \delta}=H_{3}^{*}-\frac{H_{4}^{*} \eta}{2}
\end{aligned}
$$

The derivatives are functions of the reduced wind speed $U_{\text {red }}=\frac{U}{f B}$.

This model will be used to investigate the previously described imperfections on the classic heave-pitch section model. 


\section{Imperfections and errors resulted in the heave-pitch}

\section{section model}

Consider a heave-pitch section model under a wind-tunnel investigation. During the test the model is excited with only one of its DOFs at a time (a sinusoidal heaving motion $h$ and twist motion $\alpha$ alternately).

Suppose geometric imperfections of the heave-pitch model in two ways; in the case of rotational and of heave forced vibration. In the case of the rotational excitation define the imperfection as an eccentricity $\eta \frac{B}{2}$ of the excitation axis to the c.g. of the section. This geometric imperfection could also be treated as a wrong assumption concerning the DOFs of the model. During the wind tunnel test the assumption that no heave occurs holds $(h=0)$, while the eccentricity makes the heave motion appear. The exact value of the aeroelastic forces acting on the section are the ones defined in the previous section. With the false assumption the determined derivatives contain errors which are

$$
\begin{aligned}
\Delta H_{2}^{*} & =\frac{H_{1}^{*} \eta}{2} \\
\Delta H_{3}^{*} & =\frac{H_{4}^{*} \eta}{2} \\
\Delta A_{2}^{*} & =\frac{H_{1}^{*} \eta^{2}}{4}+\frac{H_{2}^{*} \eta}{2}+\frac{A_{1}^{*} \eta}{2} \\
\Delta A_{3}^{*} & =\frac{H_{3}^{*} \eta}{2}+\frac{H_{4}^{*} \eta^{2}}{4}+\frac{A_{4}^{*} \eta}{2} .
\end{aligned}
$$

The relative errors of these derivatives are shown in figures 2 for a thin airfoil [3], [6]. The relationships of the errors in the derivatives to the imperfection are linear for some derivatives and non-linear for others, the magnitudes of the errors are in the order of the other derivatives than the one examined, and are functions of the reduced wind speed. The relative errors depend on the derivatives themselves, so they have a complex relationship with the imperfection.

The relative error in the angular speed derivative $H_{2}^{*}$ (fig. 2 (a)) for the lift force is linearly dependent on the eccentricity $\eta$, but at the zero point of the derivative the relative error produces huge values. This implies the difficulty in determining the real zero point of the derivative. The angular derivative for the lift force contains a large relative error in the precious small reduced velocity domain. Precious in the sense of an aeroelastic instability investigation.

The relative error in the angular speed derivative $A_{2}^{*}$ (fig. 2 (c)) is non-linearly dependent of the imperfection, with higher order dependency at small reduced velocities. The non-linear relationship is present at a higher value of the imperfection factor than those shown in the diagram. Due to the fact that many simplified instability analyses rely on this sole derivative the applied analysis' result is also affected by the imperfection, which leads to inaccurate flutter wind speed. One of the main purposes of a new model configuration is to diminish this error and assure reliable instability analysis results. The derivative $A_{3}^{*}($ fig. 2] (d)) is almost constantly sensitive to the imperfection at all reduced velocities, except for the small reduced velocity range where rel- ative error is non-linear and is acceptable for small eccentricity only.

For the case of the heave excitation of the model the assumption is that no rotation occurs during the test series. As the imperfection supposes a small rotation $\alpha=\frac{v}{B / 2} h$ of magnitude linear to the heave excitation, analysis steps analogue to the aforementioned ones produce errors in the heave flutter derivatives such as:

$$
\begin{aligned}
\frac{\Delta H_{1}^{*}}{H_{1}^{*}}=\frac{H_{2}^{*}}{H_{1}^{*}} \frac{2 v}{B} & \frac{\Delta H_{4}^{*}}{H_{4}^{*}}=\frac{H_{3}^{*}}{H_{4}^{*}} 2 v \\
\frac{\Delta A_{1}^{*}}{A_{1}^{*}}=\frac{A_{2}^{*}}{A_{1}^{*}} \frac{2 v}{B} & \frac{\Delta A_{4}^{*}}{A_{4}^{*}}=\frac{A_{3}^{*}}{A_{4}^{*}} 2 v
\end{aligned}
$$

The errors are linear to the imperfection factor $v$, with magnitude increasing as a function of the reduced wind speed. The model geometric size $B$ also affects the uncertainties in the derivatives $H_{1}^{*}$ and $A_{1}^{*}$ in a logical way, the larger the model size the more accurate the results produced. As the orders of $A_{1}^{*}$ and $A_{2}^{*}$, and $H_{1}^{*}$ and $H_{2}^{*}$ are the same, the error is easy to handle. On the other hand the same is not true for the remaining two derivatives as there is an order of magnitude difference between the derivative and the one that its error is related to. As there is a zero point in $H_{4}^{*}$ its relative error gets unacceptably high at reduced velocities above the zero point.

\section{Errors in the pitch-pitch section model}

Similarly to the heave-pitch section model the determination of the derivatives of the pitch-pitch section model in a windtunnel investigation is to be performed in two steps according to the two degrees of freedom. The section model is excited by sinusoidal rotations in its DOFs alternately. In the case of an oscillating rotation around the windward rotation point the assumption of having no rotation around the leeward axis holds (case of excitation in $\gamma$ and $\delta=0$ ). The recorded lift force and torsion moment around the axis in point $\mathrm{G}$ give the derivatives with index $\gamma$. The same steps apply for the determination of derivatives corresponding to the rotation around the axis in point $\mathrm{D}$ and result in derivatives with index $\delta$.

The investigation of the errors in the measured derivatives due to the imperfection in the pitch-pitch model leads to the followings. Let the imperfection be defined as an unexpected movement of the fixed point described by a rotation $\delta=\lambda \gamma$ in the non-excited DOF. The errors of the derivatives shall be:

$$
\begin{array}{rlrl}
\Delta A_{2 \gamma}^{*} & =\lambda\left(A_{2 \delta}^{*}+\eta H_{2 \delta}^{*}\right) & \Delta A_{3 \gamma}^{*}=\lambda\left(A_{3 \delta}^{*}+\eta H_{3 \delta}^{*}\right) \\
\Delta H_{2 \gamma}^{*}=\lambda H_{2 \delta}^{*} & \Delta H_{3 \gamma}^{*}=\lambda H_{3 \delta}^{*}
\end{array}
$$

Similar relations can be concluded for the other pitch excitation test series, with an independent $\lambda$ factor for this case. The errors in the derivatives are given as:

$$
\begin{array}{rlrl}
\Delta A_{2 \delta}^{*} & =\lambda\left(A_{2 \gamma}^{*}-\eta H_{2 \gamma}^{*}\right) & \Delta A_{3 \delta}^{*}=\lambda\left(A_{3 \gamma}^{*}-\eta H_{3 \gamma}^{*}\right) \\
\Delta H_{2 \delta}^{*}=\lambda H_{2 \gamma}^{*} & \Delta H_{3 \delta}^{*}=\lambda H_{3 \gamma}^{*}
\end{array}
$$




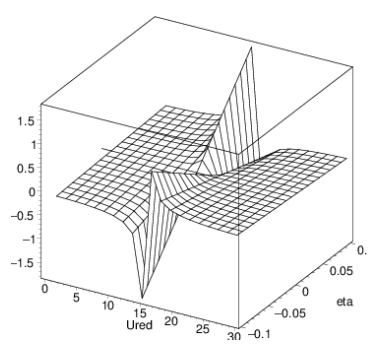

(a) Relative error in $H_{2}^{*}$

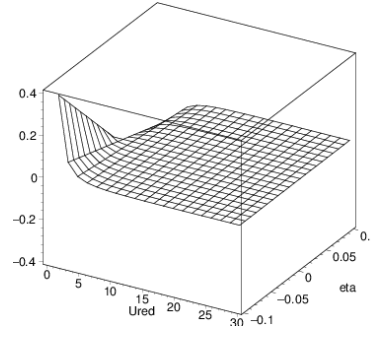

(b) Relative error in $H_{3}^{*}$

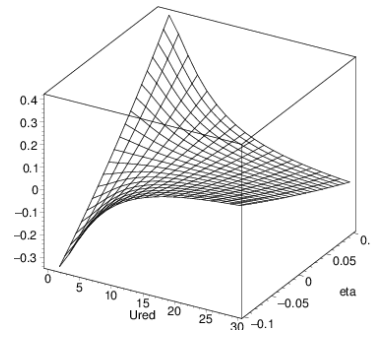

(c) Relative error in $A_{2}^{*}$

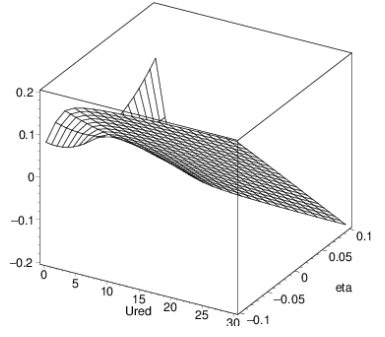

(d) Relative error in $A_{3}^{*}$

Fig. 2. Relative errors in $\frac{\Delta H_{i}^{*}}{H_{i}^{*}}$ and $\frac{\Delta A_{i}^{*}}{A_{i}^{*}}(i=2,3)$ due to imperfect eccentricity $\eta$ (case of an thin airfoil)

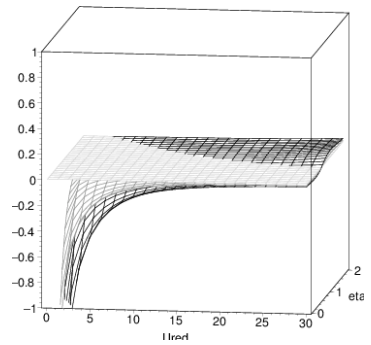

(a) Relative error in derivative $A_{2 \gamma}^{*}$

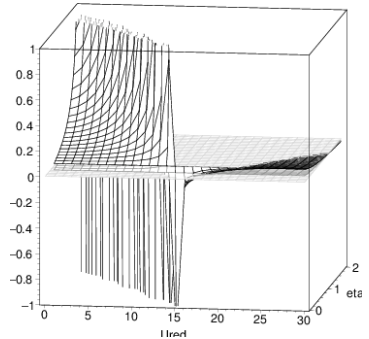

(b) Relative error in derivative $H_{2 \gamma}^{*}$

Fig. 3. Relative errors in angular speed derivatives with imperfection factor magnitude of $\lambda=0,0.05,0.1$ (light grey, mid-grey, black, resp.)

Without presenting diagrams of the relative errors in all derivatives the remarks are the following for the case of a thin airfoil. The relative errors in the derivatives $A_{2 i}^{*}(i=\gamma, \delta$ ) (fig. 3 (a)) are only present in the region of small reduced wind speeds. These errors decrease with increasing eccentricity $\eta$ of the rotation axes. This means that the derivative sensibility to the imperfection can be weakened by spacing the two rotation points. Derivative $A_{3 \gamma}^{*}$ is highly contaminated by the imperfection factor $\lambda$ at the precious small reduced velocities, which error is not present in the derivative $A_{3 \delta}^{*}$. The same applies to the derivatives $H_{3 \gamma}^{*}$ and $H_{3 \delta}^{*}$ for the lift force. Angular speed derivatives contributing to the lift force contain high errors in the lower and higher reduced velocity ranges for $H_{2 \delta}^{*}$ and $H_{2 \gamma}^{*}$ (fig. 3 (b)), respectively.

\subsection{Determination of the derivatives defined to the centre of gravity}

The derivatives determined on the pitch-pitch section model are converted into the derivatives defined to the centre of gravity of the section using the equations (3) and (4). The relative errors in the latter derivatives were determined for a thin airfoil, using the theoretically defined derivatives of Theodorsen [7], and the derivatives of the extradosed bridge of the motorway M43 in Hungary [4]. During the analysis we assumed that the two phases of the wind tunnel tests and their imperfections are independent from each other. The derivatives of the bluff section were fitted on the data measured in a wind tunnel on a heavepitch section model and are treated hereafter as the derivatives without imperfections, see figure 5 .

The figures 4 and 5 present the perfect and imperfect (erro- neous) derivatives with different supposed imperfection factors in the wind tunnel test $(\lambda=0,0.05,0.1)$. Although an imperfection of $10 \%$ is relatively high, it is used as a demonstration in the present theoretical investigation. It can be observed that the same motion derivatives corresponding to the torsion moment and the lift force have similar diagrams (e.g. $H_{1}^{*}-A_{1}^{*}$, fig. 4 (a) 4 (b) for the case on the thin airfoil, fig. 5 (a) -5 (b) for the bluff section). The derivatives $H_{3}^{*}$ and $A_{3}^{*}$ do not depend on neither the imperfection factor $\lambda$ nor the eccentricity $\eta$. The absolute errors in the derivatives $H_{2}^{*}$ and $A_{2}^{*}$ (fig. 4 (c) and 5 (c)) increase with the supposed imperfection and increase with the applied eccentricity for the airfoil but decrease with it for the bluff section. All errors in the other derivatives show a dependency on the reduced velocity (e.g. 4(d) and 5(d)). These errors can be compensated by increasing the applied eccentricity $\eta$, in other words by spacing the two rotational axes.

Concerning the relative errors in the derivatives (figures 6 and 7) the followings are concluded. Relative errors in some derivatives show dependency on the reduced wind speed, others show sensibility to the applied eccentricity depending on the reduced velocity, and others show improvements with increasing eccentricity. The heave speed derivatives $H_{1}^{*}$ and $A_{1}^{*}$ (fig. 6 (a) and 7 (b)) include errors of great magnitude at almost all reduced velocities, which errors can be diminished by increasing the applied eccentricity. The relative errors in the heave derivatives show similar behaviour, but the errors stay unacceptable at both small and high eccentricities. This causes no huge practical issues as these derivatives are in several cases omitted from the flutter analysis. The angular speed derivatives $H_{2}^{*}$ (fig.77(a)) and 


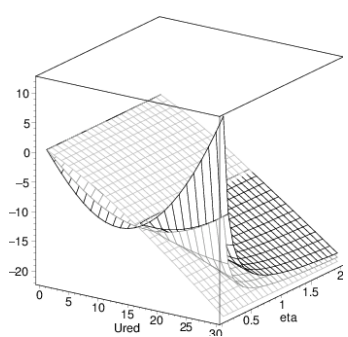

(a) $H_{1}^{*}$

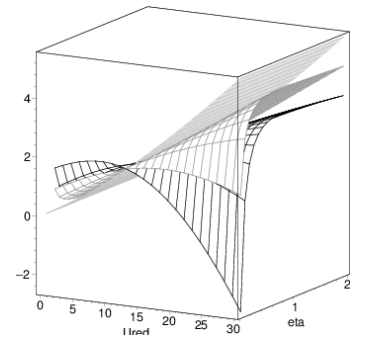

(b) $A_{1}^{*}$

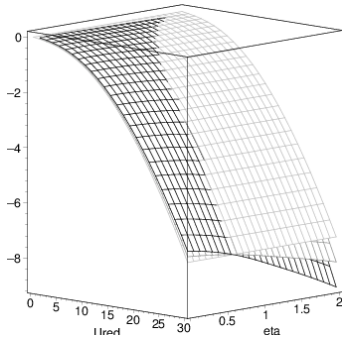

(c) $A_{2}^{*}$

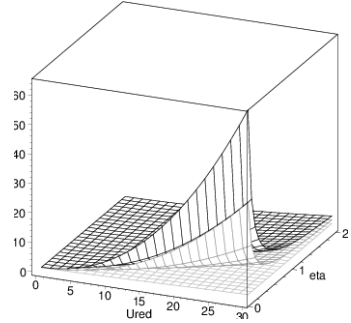

(d) $A_{4}^{*}$

Fig. 4. Thin airfoil derivatives due to the imperfection factor $\lambda$. Light grey: without imperfection $(\lambda=0)$, mid-grey: $\lambda=0.05$, black: $\lambda=0.10$

$A_{2}^{*}$ (fig. 6/(b) and 7 (c)) are contaminated with errors linearly dependent on the eccentricity, and the determination of their zero becomes inaccurate. The relative errors included in the angular derivatives show strange relations. The relative error in the derivative $H_{3}^{*}$ for the lift force decreases exponentially as a function of the reduced velocity, even if the eccentricity increases it. This gives acceptable measures of this derivative at all reduced velocities but the very small ones. The angular derivative $A_{3}^{*}$ for the torsional moment seems to be practically insensible to the eccentricity and the supposed imperfections. The heave derivatives $H_{4}^{*}$ and $A_{4}^{*}$ show relative errors with magnitude of great significance, the determination of these derivatives with the pitch-pitch model is questionable.

The relative errors are functions of the supposed imperfections and it seems that the model size has no effect on them. But as the size of the model increases, or as the applied eccentricity increases the imperfection diminishes, due to the easier geometric manoeuvrability.

\section{Sensitivity of the flutter speed to the model imperfec- tions}

The study investigated also the effect of the imperfections on the critical wind velocity, referred to as flutter speed $U_{F}$. The complex eigenvalue analysis [5] of an undamped two degreeof-freedom cross section was used in the determination of the flutter speed. The effect is represented as the ratio of the flutter speed resulted with the erroneous derivatives to the one with no errors. The most unfavourable sign combination of the eccentricities and the imperfections were used to obtain the representable values. Hereafter the relative mass $\mu$ is the ratio of the mass of the cross section to the mass of the circumscribed air mass, the relative torsional inertia radii $r_{\alpha}$ is the ratio of the torsional inertial radius to the half width, and $\epsilon$ represents the ratio of the considered torsional to the heave eigenfrequencies.

The erroneous flutter speed on the heave-pitch ( $h$ and $\alpha$ ) model is influenced by the width $B$ of the section. The figure 8 (a) presents the case of the heave-pitch model of a thin airfoil with the torsional imperfections $v$ and the eccentricity $\eta$ of the rotational axis to the centre of gravity. It is concluded, that although the relative mass $\mu$ compensates the error, the latter reaches quite important values even at high eigenfrequency ratios $\epsilon$.
The figure 8 (b) shows the results of a similar analysis this time based on the pitch-pitch model with the torsional imperfections $\lambda_{\delta}$ and $\lambda_{\gamma}$ for the two rotational degrees, resp. The variation of the flutter speed is represented as a function of the applied $\eta$ eccentricity. The error seems to highly depend on the eccentricity and has an extremum at a singular value of it. The similar diagram shown on fig. 9 is resulted for the case of the bluff section.

The coupled flutter phenomenon of a bridge cross section appears as an undamped rotation around an eccentric quasi rotational axis. The phase shift of the vertical and angular harmonic motion causes the vertical movement of this quasi rotational axis, that is why we have denote it "quasi" (not to be confused with the body, nor the space rotational axis). One could assume that the optimal eccentricity, when the flutter speed error is minimum, coincides with the quasi rotation point. This coincidence could be used in the wind tunnel investigation by preliminarily estimating the required eccentricity of the set-up based on experiences in hope of minimise the errors treated in this paper. Unfortunately the analyses showed that the optimal eccentricities for the presented configurations were at around 1.3-1.8 times the eccentricity of the quasi rotational point.

\section{Conclusions}

The analysis of the effects of the supposed (model or geometric) imperfections and eccentricity of a heave-pitch section model on the derivatives was studied. The discussed errors increase the uncertainties of determination of the zeros of the derivatives. The imperfections appear as high relative errors in several derivatives $\left(H_{1}^{*}, H_{3}^{*}, A_{2}^{*}\right.$ and $\left.A_{4}^{*}\right)$ in the precious range of small reduced velocities, or in other ranges for $A_{1}^{*}$ and $A_{3}^{*}$.

A wind tunnel test carried out on a pitch-pitch section model yields newly defined derivatives, from which those defined to the centre of gravity of the section are calculated. On this model the impact of the supposed imperfections can be decreased for some specific derivatives $\left(H_{2}^{*}, H_{4}^{*}, A_{1}^{*}\right.$ and $\left.A_{4}^{*}\right)$. The derivatives $H_{3}^{*}$ and $A_{3}^{*}$ show to be practically insensible to the imperfections. The application of an adequate eccentricity of the forced rotational points seems to moderate the effects of the rotational imperfections on the derivatives $H_{1}^{*}$ and $A_{2}^{*}$. 


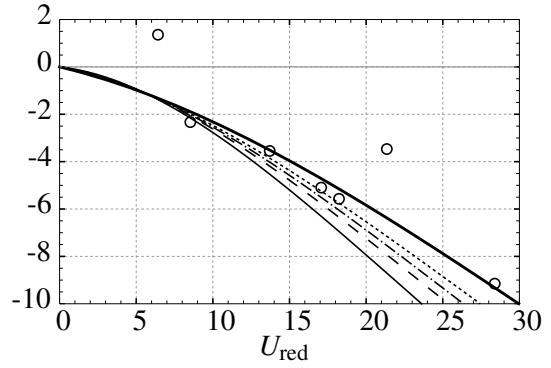

(a) $H_{1}^{*}$

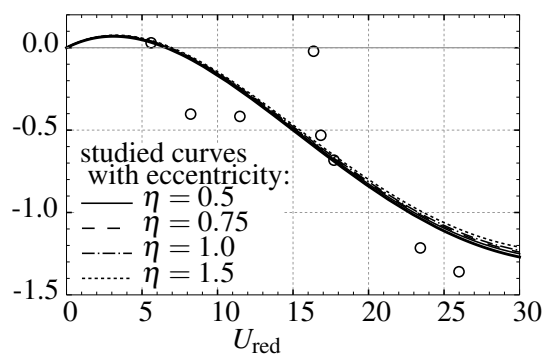

(c) $A_{2}^{*}$

Fig. 5. M43 derivatives: tick line $=$ derivative fitted on measured data and referred to as without imperfections, thin lines $=$ studied derivatives supposing

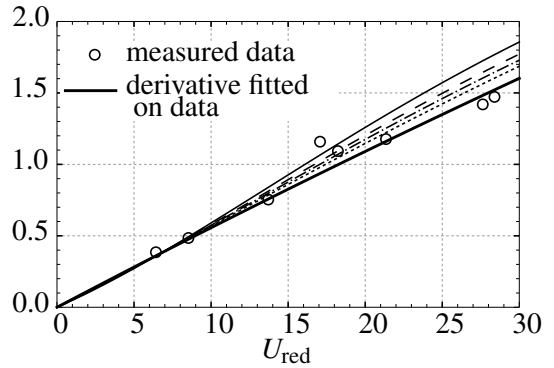

(b) $A_{1}^{*}$

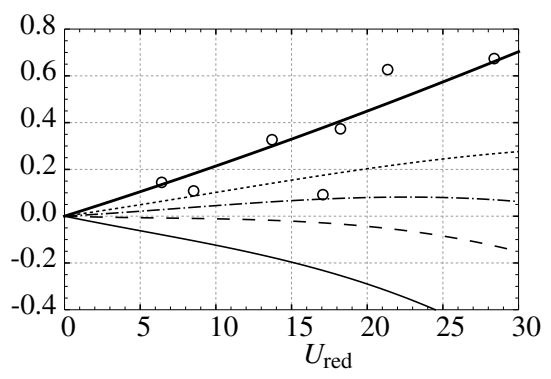

(d) $A_{4}^{*}$

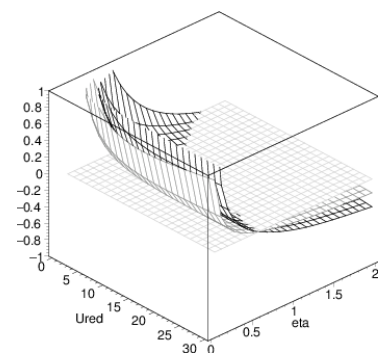

(a) Relative error in derivative $A_{1}^{*}$

Fig. 6. $\frac{\Delta A_{i}^{*}(\lambda)}{A_{i}^{*}}$ relative errors in the thin airfoil derivatives as a function of the applied eccentricity and the imperfection factor $\lambda$. Light grey: without imper-

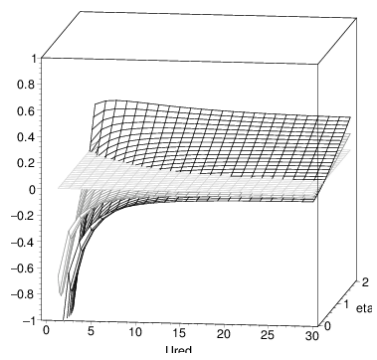

(b) Relative error in derivative $A_{2}^{*}$

fection $(\lambda=0)$, mid-grey: $\lambda=0.05$, black: $\lambda=0.10$

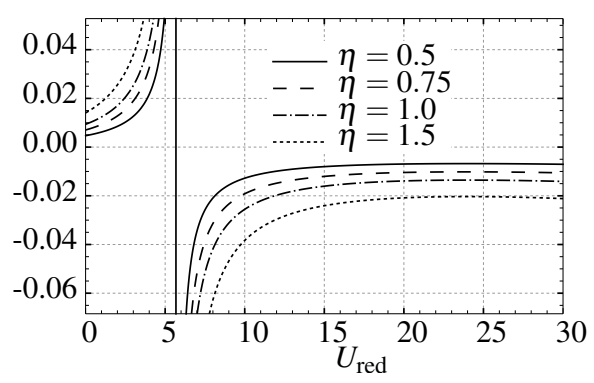

(a) $H_{2}^{*}$

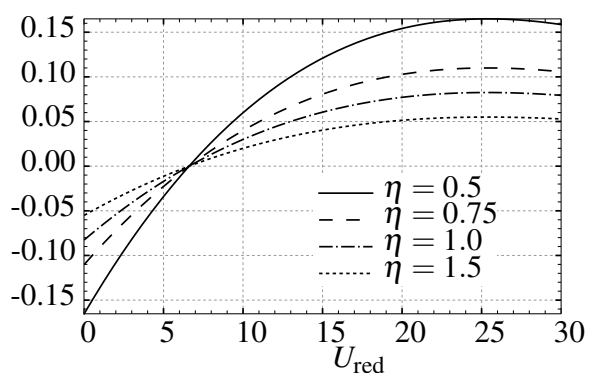

(b) $A_{1}^{*}$

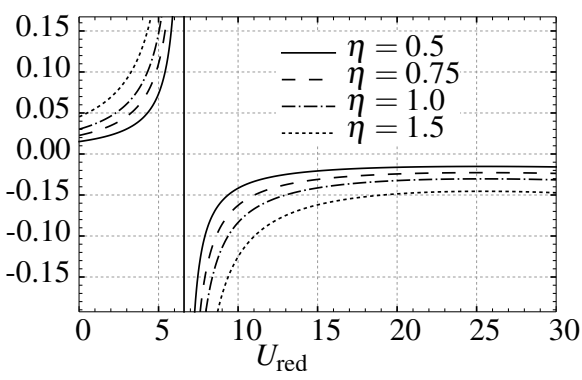

(c) $A_{2}^{*}$

Fig. 7. $\frac{\Delta A_{i}^{*}(\lambda)}{A_{i}^{*}}$ relative errors in the M43 derivatives as a function of the applied eccentricity $\eta$ and the imperfection factor $\lambda=0.05$ 


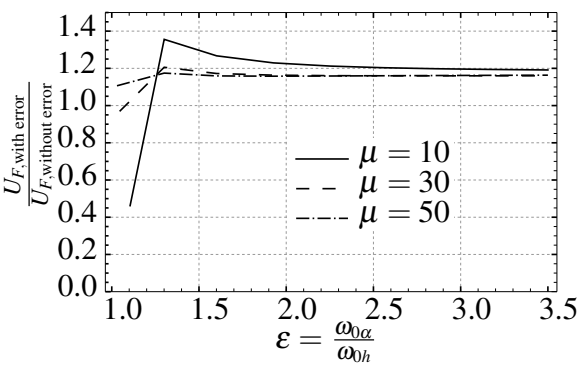

(a) Heave-pitch $(h-\alpha)$ model, $B=1 \mathrm{~m}, \eta=0.1$, $v=-0.1$

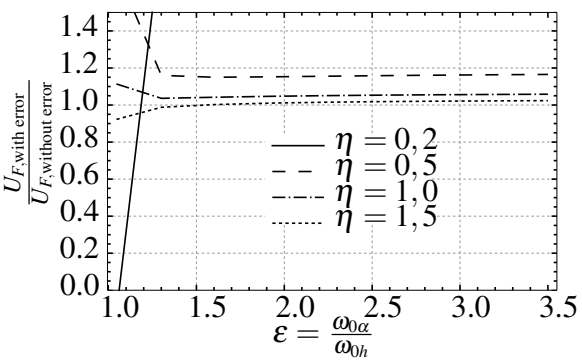

(b) Pitch-pitch $(\gamma-\delta)$ model, $\mu=30$, $\lambda_{\gamma}=-\lambda_{\delta}=-0.10$

Fig. 8. Effect of the imperfection on the flutter speed. Common parameters: thin airfoil, $r_{\alpha}=0.5$

It seems that an optimum of the applied eccentricity for derivatives $A_{1}^{*}$ and $A_{2}^{*}$ exists where these derivatives contain the least error. The heave derivative $A_{4}^{*}$ contains an error of great magnitude, but maybe less at the same value of eccentricity. The relative errors in the derivatives $H_{1}^{*}, H_{3}^{*}$ and $H_{4}^{*}$ for the aeroelastic lift force tend to diminish by augmenting the eccentricity of the rotation points. The inverse is concluded for the derivative $H_{2}^{*}$, where the error diminishes as the rotation points get closer to each other.

The investigation of the effect of the imperfections on the changes of the flutter speed showed the existence of an optimal eccentricity of the pitch-pitch model. Unfortunately this optimal eccentricity did not coincide with the quasi rotational point at flutter and thus the eccentricity to be applied cannot be preliminarily estimated by the latter to minimise the error of the flutter speed.

The applicability of the presented set-up requires the further investigation of the effects on the errors present in the wind tunnel tests.

\section{Acknowledgement}

This work is connected to the scientific program of the "Development of quality-oriented and harmonized $\mathrm{R}+\mathrm{D}+\mathrm{I}$ strategy and functional model at BME" project. This project is supported by the New Széchenyi Plan (Project ID: TÁMOP4.2.1/B-09/1/KMR-2010-0002).

\section{References}

1 Billah K., Scanlan R., Resonance, Tacoma Narrows bridge failure, and undergraduate physics textbooks, American Journal of Physics 59 (1991), 118124 , DOI $10.1119 / 1.16590$.
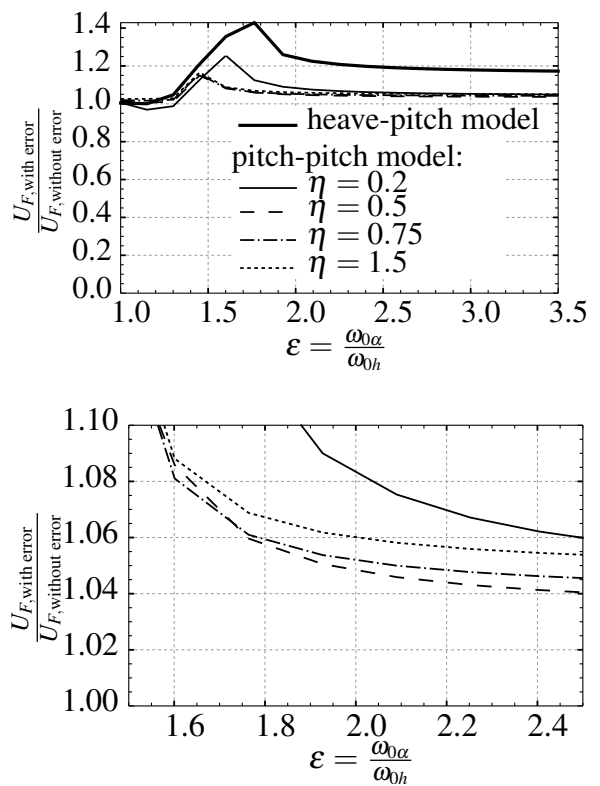

Fig. 9. Effect of the imperfection on the flutter speed. M43 derivatives, $r_{\alpha}=$ 0.75, $\mu=30$, width $B=30 \mathrm{~m}$. Imperfections considered: case of heave-pitch model $v=-0.05, \eta=0.05$, case of pitch-pitch model $\lambda_{\delta}=-\lambda_{\gamma}=0.05$

2 Caracoglia L, Feasibility assessment of a leading-edge-flutter wind power generator, Journal of Wind Engineering and Industrial Aerodynamics 98 (2010), no. 10-11, 679-686, DOI 10.1016/j.jweia.2010.04.006.

3 Cremona C. a. F., Comportement au vent des ponts (Presses de l'Ecole Nationale des Ponts et Chaussées (ENPC), ed.), Association Française de Génie Civil (AFGC), 2002.

4 Hunyadi M, Flutter analysis of an extradosed bridge in Hungary, 5th European \& African Conference on wind Engineering (Firenze, Italy, July 19, 2009), 5th European \& African Conference on wind Engineering: Conference Proceedings (Borri C, D'Asdia P, Spinelli P, eds.), Firenze University Press, 2009, pp. 357-360. Full paper on annexed CD.

5 KIöppel K, Thiele F, Modellversuche im Windkanal zur Bemessung von Brücken gegen die Gefahr winderregter Schwingungen, Der Stahlbau 36 (1967), no. 12, 353-365.

6 Simiu E, Scanlan R., Wind Effects on Structures: Fundamentals and Applications to Design, Wiley, 1996.

7 Theodorsen T, General theory of aerodynamic instability and the mechanism of flutter, 496, National Advisory Committee for Aeronautics, 1935.

8 Szabó G, Györgyi J, Numerical simulation of the flutter performance of different generic bridge cross sections, Periodica Polytechnica, Civil Engineering 55 (2011), no. 2, 137-146. 